The Astrophysical Journal, 265:18-25, 1983 February 1

(C) 1983. The American Astronomical Society. All rights reserved. Printed in U.S.A.

\title{
INFRARED/OPTICAL ENERGY DISTRIBUTIONS OF HIGH-REDSHIFT QUASARS
}

\author{
B. T. Soifer, G. Neugebauer, J. B. Oke, K. Matthews, and J. H. Lacy \\ Palomar Observatory, California Institute of Technology \\ Received 1982 April 26; accepted 1982 July 30
}

\begin{abstract}
Measurements at 1.2, 1.6, and $2.2 \mu \mathrm{m}$ have been combined with visual spectrophotometry of 21 quasars having redshifts $z \geq 2.66$. The primary result of this study is that the rest frame visual/ultraviolet continua of the high-redshift quasars are well described by a sum of a power-law continuum with slope $\sim-0.4$ and a $3000 \AA$ bump. The rest frame visual/ultraviolet continua of these quasars are quite similar to that of $3 \mathrm{C} 273$, the archetype of low-redshift quasars. There do not appear to be any significant visual/ultraviolet properties distinguishing high-redshift quasars selected via visual or radio techniques.
\end{abstract}

Subject headings: quasars - spectrophotometry

\section{INTRODUCTION}

Understanding of the emission mechanisms and underlying energy sources of the visual and ultraviolet continua in quasars has long been an important goal of quasar observations. The discovery of a significant number of moderately bright high-redshift quasars allows a detailed study of the rest frame ultraviolet and visual continua to be done by observing these objects at visual and near-infrared wavelengths. In this paper we report the results of such a study for 21 quasars having redshifts $z \geq 2.66$.

\section{THE SAMPLE}

The objects included in this study were all selected to be moderately bright $\left(m_{v}<20 \mathrm{mag}\right)$ quasars with redshifts sufficiently large to bring the Lyman continuum discontinuity into the visual wavelength range. The objects are listed in Table 1. A discussion of the results of the study of the Lyman discontinuity in this sample is found in Oke and Korycansky (1982).

Fourteen objects in the present sample were discovered originally in radio surveys. The remaining seven were discovered in visual surveys; five of these were discovered in the University of Michigan objective prism survey (MacAlpine and Lewis 1978; MacAlpine, Smith, and Lewis 1977). Positions and redshifts for all the objects in this study with the exception of 1320-10 (POX 175; Kunth, Sargent, and Kowal 1981) are found in the catalog of Hewitt and Burbidge (1980). It is clear that the sample observed is not homogeneous; neither the radio nor visually selected samples obey well-defined selection criteria.

\section{OBSERVATIONS}

All the new observations reported here were obtained on the Hale $5.08 \mathrm{~m}$ telescope at Palomar Mountain. The visual observations at observed wavelengths between $3200 \AA$ and $10,000 \AA$ (rest wavelengths between $\sim 800 \AA$ and $2500 \AA$ ) were obtained with the multi- channel spectrometer with bandpasses of $80 \AA$ below $5600 \AA$ and $160 \AA$ above $5600 \AA$. These data were reported in Oke and Korycansky (1982). In the case of PKS 0528-253, multichannel observations were not available, and published observations (Smith, Jura, and Margon 1979) were used in the visual range. Because of the large air mass and small aperture (2".8) used for the observations of PKS 0528 - 253, the absolute calibration has a large uncertainty, and the relative calibration may suffer from differential refraction effects (Smith, private communication). The infrared observations in the $1.25 \mu \mathrm{m}, 1.65 \mu \mathrm{m}$, and $2.2 \mu \mathrm{m}$ photometric bands were obtained with a solid nitrogen-cooled InSb detector. The diameter of the focal-plane aperture for all the infrared observations was $5^{\prime \prime}$.

The continuum flux densities in the three infrared bands $(1.25 \mu \mathrm{m}, 1.65 \mu \mathrm{m}$, and $2.2 \mu \mathrm{m})$ are given in Table 1 for each quasar; at a mean redshift of 3 these correspond to rest frame wavelengths of $\sim 0.31 \mu \mathrm{m}$, $\sim 0.41 \mu \mathrm{m}$, and $\sim 0.55 \mu \mathrm{m}$. The observations have been corrected for emission lines in the photometric bands as discussed below, and the magnitudes of these corrections are given in Table 2 . The flux density at a rest wavelength of $0.174 \mu \mathrm{m}$ obtained from the multichannel observations is also included in Table 1. In several cases infrared and visual photometry were obtained on several nights for the same object. Except as noted below, the observations agreed within their statistical uncertainties for the objects for which multiple observations were obtained, and the flux densities reported represent the average of all observations, weighted by their uncertainties.

In two cases, $0758+12$, and $2351-154$, the infrared photometry at one or more wavelengths suggests significant variability; in both cases there is evidence for color changes as well. The dates of observations and observed magnitudes at $1.2,1.6$, and $2.2 \mu \mathrm{m}$ of $0758+12$ and 2351-154 are given in Table 3. For $0758+12$ the data at $2.2 \mu \mathrm{m}$ indicate variability at the $99 \%$ confidence 
TABLE 1

Continuum Flux Densities of High-Redshift Quasars

\begin{tabular}{|c|c|c|c|c|c|c|c|c|}
\hline \multirow[b]{3}{*}{ ОВЈеСт } & \multirow{3}{*}{$\begin{array}{l}\text { OTHER } \\
\text { NAME }\end{array}$} & \multirow[b]{3}{*}{$z$} & \multicolumn{4}{|c|}{$\log f_{v}(\mathrm{mJy})$} & \multirow{2}{*}{\multicolumn{2}{|c|}{ OBSERVATION DATES }} \\
\hline & & & \multicolumn{4}{|c|}{$\lambda_{0}(\mu \mathrm{m})$} & & \\
\hline & & & 0.174 & $\sim 0.31$ & $\sim 0.41$ & $\sim 0.55$ & Visual & Infrared \\
\hline $0054-00$ & PKS & 2.795 & -1.10 & $-1.00 \pm 0.04$ & $-1.02 \pm 0.06$ & $-1.21 \pm 0.12$ & 1977 Aug & 1980 Aug, 1981 Nov \\
\hline $0100+13$ & PHL 957 & 2.690 & -0.04 & $+0.09 \pm 0.02$ & $+0.06 \pm 0.02$ & $-0.07 \pm 0.02$ & 1970 Oct & 1976 Oct, 1979 Jul, 1979 Nov, 1981 Nov \\
\hline $0143-015$ & UM 366 & 3.14 & -0.47 & $-0.35 \pm 0.05$ & $-0.31 \pm 0.03$ & $-0.35 \pm 0.04$ & 1980 Sep & 1980 Oct \\
\hline $0143-010$ & UM 368 & 3.16 & -1.17 & $-0.94 \pm 0.12$ & $-0.85 \pm 0.05$ & $-0.72 \pm 0.06$ & $1980 \mathrm{Sep}, 1980 \mathrm{Dec}$ & 1980 Oct \\
\hline $0146+017$ & UM 141 & 2.91 & -0.44 & $-0.33 \pm 0.04$ & $-0.24 \pm 0.03$ & $-0.26 \pm 0.04$ & $1980 \mathrm{Dec}$ & 1980 Oct \\
\hline $0154+045$ & UM 148 & 2.99 & -0.58 & $-0.36 \pm 0.05$ & $-0.27 \pm 0.03$ & $-0.28 \pm 0.04$ & $1981 \mathrm{Jan}$ & 1980 Oct \\
\hline $0207+003$ & UM 402 & 2.84 & -0.22 & $-0.04 \pm 0.05$ & $-0.05 \pm 0.03$ & $-0.08 \pm 0.05$ & $1980 \mathrm{Dec}$ & 1980 Oct \\
\hline $0528-25$ & PKS & 2.765 & -0.52 & $-0.43 \pm 0.08$ & $-0.28 \pm 0.04$ & $-0.36 \pm 0.04$ & & $1980 \mathrm{Feb}$ \\
\hline $0642+449$ & OH 471 & 3.402 & -0.77 & $-0.53 \pm 0.03$ & $-0.51 \pm 0.03$ & $-0.50 \pm 0.02$ & 1973 Apr, 1973 Dec & 1980 Feb, 1981 Nov \\
\hline $0758+12$ & MC 5 & 2.660 & -0.94 & $-0.93 \pm 0.03$ & $-1.03 \pm 0.03$ & $-1.17 \pm 0.04$ & $1978 \mathrm{Feb}$ & $1980 \mathrm{Feb}, 1981$ Oct, $1981 \mathrm{Nov}$ \\
\hline $0805+046$ & $4 C 05.34$ & 2.877 & -0.66 & $-0.50 \pm 0.03$ & $-0.43 \pm 0.03$ & $-0.55 \pm 0.03$ & 1978 Dec, 1979 Oct & 1980 Feb, 1981 Nov \\
\hline $0830+115$ & MC 5 & 2.974 & -0.77 & $-0.71 \pm 0.04$ & $-0.67 \pm 0.03$ & $-0.78 \pm 0.04$ & 1977 Feb & $1980 \mathrm{Feb}, 1981 \mathrm{Nov}$ \\
\hline $0938+119$ & MC 5 & 3.190 & -1.31 & $-1.06 \pm 0.06$ & $-1.01 \pm 0.04$ & $-1.20 \pm 0.09$ & 1977 Feb, 1977 Dec & 1980 Feb, 1981 Nov \\
\hline $0941+261$ & OK 270 & 2.910 & -0.87 & $-1.04 \pm 0.20$ & $-0.72 \pm 0.04$ & $-0.72 \pm 0.06$ & 1977 Feb & $1980 \mathrm{Feb}$ \\
\hline $1004+14$ & OL 108.1 & 2.707 & -1.00 & $-0.74 \pm 0.07$ & $-0.71 \pm 0.03$ & $-0.77 \pm 0.04$ & 1977 Feb, 1977 Dec, 1978 Feb & $1980 \mathrm{Feb}$ \\
\hline $1320-10$ & POX 175 & 3.14 & & $\ldots$ & $-0.35 \pm 0.04$ & $-0.44 \pm 0.04$ & & $1980 \mathrm{Feb}$ \\
\hline $1402+044$ & PKS & 3.202 & -1.22 & & $-1.04 \pm 0.08$ & $-0.97 \pm 0.10$ & $1978 \mathrm{Jul}$ & $1980 \mathrm{Feb}, 1980 \mathrm{Mar}$ \\
\hline $1442+101$ & OQ 172 & 3.53 & -0.49 & $-0.38 \pm 0.07$ & $-0.47 \pm 0.04$ & $-0.43 \pm 0.05$ & $1973 \mathrm{Jul}$ & $1980 \mathrm{Jul}$ \\
\hline $2126-158$ & PKS & 3.270 & -0.11 & $-0.00 \pm 0.02$ & $+0.05 \pm 0.02$ & $-0.04 \pm 0.02$ & $1978 \mathrm{Jul}$ & 1978 Jul, 1981 Aug, 1981 Nov \\
\hline $2256+017$ & PKS & 2.663 & -0.97 & $-0.74 \pm 0.03$ & $-0.80 \pm 0.02$ & $-0.92 \pm 0.04$ & $1978 \mathrm{Dec}$ & $1980 \mathrm{Jul}, 1981$ Oct, 1981 Nov \\
\hline $2351-154$ & OZ 187 & 2.665 & $\ldots$ & $-0.30 \pm 0.02$ & $-0.27 \pm 0.02$ & $-0.30 \pm 0.02$ & $\ldots$ & 1980 Jul, 1981 Oct, 1981 Nov \\
\hline
\end{tabular}


TABLE 2

Corrections to Observed Flux Densities FOR EMISSION LINES

\begin{tabular}{|c|c|c|c|}
\hline \multirow[b]{3}{*}{ ОвJест } & \multicolumn{3}{|c|}{ CORRECTION $^{a}$} \\
\hline & \multicolumn{3}{|c|}{ WAVELENGTH $(\mu \mathrm{m})$} \\
\hline & 1.25 & 1.65 & 2.2 \\
\hline $0100+13 \ldots \ldots$ & $\ldots$ & 0.04 & $0.06^{\mathrm{b}}$ \\
\hline $0143-015 \ldots \ldots$ & $\ldots$ & $\ldots$ & $0.03^{\mathrm{c}}$ \\
\hline $0143-010 \ldots \ldots$ & 0.02 & $\ldots$ & $0.04^{\mathrm{c}}$ \\
\hline $0528-25 \ldots \ldots$ & $\ldots$ & 0.01 & \\
\hline $0642+449 \ldots \ldots$ & $\ldots$ & $\ldots$ & $0.07^{\mathrm{c}}$ \\
\hline $0758+12 \ldots \ldots$ & $\ldots$ & 0.05 & $0.12^{b}$ \\
\hline $0938+119 \ldots \ldots$ & 0.02 & $\ldots$ & $0.05^{\mathrm{c}}$ \\
\hline $1004+14 \ldots \ldots$ & $\ldots$ & 0.03 & $0.07^{b}$ \\
\hline $1320-10 \ldots \ldots$ & $\ldots$ & $\ldots$ & $0.03^{\mathrm{c}}$ \\
\hline $1402+044 \ldots \ldots$ & $\ldots$ & $\ldots$ & $0.05^{\mathrm{c}}$ \\
\hline $1442+101 \ldots \ldots$ & $\ldots$ & $\ldots$ & $0.07^{\mathrm{c}}$ \\
\hline $2126-158 \ldots \ldots$ & $\ldots$ & $\ldots$ & $0.01^{\mathrm{c}}$ \\
\hline $2256+017 \ldots \ldots$ & $\ldots$ & 0.06 & $0.12^{\mathrm{b}}$ \\
\hline $2351-154 \ldots \ldots$ & $\ldots$ & 0.05 & $0.12^{b}$ \\
\hline
\end{tabular}

level, while the data at other wavelengths do not show evidence for such variability. For $2351-154$ the data at all three wavelengths show strong evidence for variability, with a total variation of $1.5 \mathrm{mag}$ at $2.2 \mu \mathrm{m}$. Indeed it is clear that this quasar varied by $\sim 30 \%$ over the one month period of 1981 October/November. In this case there is marginal evidence for a color change with the variation, the $1.6-2.2 \mu \mathrm{m}$ color changing by $0.4 \pm 0.2$ mag between 1980 July and 1981 November.

The infrared continuum observations are affected to some extent by inclusion of strong emission lines in the bandpasses of the observations. The strongest lines in the spectra of quasars that affect the continuum fluxes are $\mathrm{H} \alpha, \mathrm{H} \beta$, [O III] $\lambda \lambda 4959,5007$, and $\mathrm{Mg}$ II $\lambda 2800$. Lines weaker than these have small enough equivalent widths to make a negligible individual contribution to the measured continuum level. Of course the sum of weak lines may serve to raise the overall continuum level by a significant amount, but no correction was made for this effect.

For the $\mathrm{H} \alpha, \mathrm{H} \beta$, and [O III] lines, corrections were applied to the observed flux densities assuming the rest frame equivalent width of each line was equal to the average rest frame equivalent width observed in lower redshift quasars (Neugebauer et al. 1979, hereafter NOBM; Soifer et al. 1981; Puetter et al. 1981). The work of Soifer et al. and Puetter et al. suggests no strong dependence of hydrogen line strengths on redshift (and hence luminosity). No luminosity dependence has been reported for the strength of [O III]. For $\mathrm{H} \alpha, \mathrm{H} \beta$, and [O III], the rest frame equivalent widths were taken to be 300,100 , and $50 \AA$ respectively. For $\mathrm{H} \alpha$ and $\mathrm{H} \beta$ the line width was assumed to be $10,000 \mathrm{~km} \mathrm{~s}^{-1}$ (full width at zero intensity), while the [O $\mathrm{mI}]$ lines were assumed to be quite narrow. A correction was made for contributions of the $\mathrm{Mg}$ II line in the $1.2 \mu \mathrm{m}$ flux density for quasars having redshifts $z>3.1$. The maximum correction to the continuum flux for those quasars affected by the line is expected to be less than $10 \%$. This correction is made uncertain by the possible luminosity dependence of the equivalent width of $\mathrm{Mg}$ II noted by Baldwin et al. (1978). Because of this, a correction was applied only for two quasars, whose rest frame $\mathrm{Mg}$ II $\lambda 2800$ equivalent width is predicted, on the basis of results of Baldwin et al., to be $\gtrsim 30 \AA$.

No attempt has been made to estimate an uncertainty associated with the corrections in Table 2 since the corrections were made for an average quasar emissionline spectrum, and there is significant variation among the quasars. Continuum flux densities for wavelengths longer than $\mathrm{Ly} \alpha$ from the multichannel spectrophotometry and infrared photometry corrected for emissionline contributions are shown in Figure 1. Where variability is suggested, the energy distribution plotted in Figure 1 is that at a single epoch, with the visual and infrared data separated by the minimum time available. For comparison, the energy distribution of 3C 273 (Boggess et al. 1979) over the same rest frame wavelength range is also shown.

TABLE 3

Photometry of SusPected Variable Quasars

\begin{tabular}{ccccc}
\hline \hline Object & Date & $1.2 \mu \mathrm{m}$ & $1.6 \mu \mathrm{m}$ & $2.2 \mu \mathrm{m}$ \\
\hline $0758+12 \ldots \ldots \ldots \ldots$ & 1980 Feb & $>17.44$ & $17.49 \pm 0.18$ & $17.26 \pm 0.15$ \\
& 1981 Oct & $17.77 \pm 0.11$ & $17.34 \pm 0.10$ & $16.66 \pm 0.14$ \\
& 1981 Nov & $17.77 \pm 0.12$ & $17.53 \pm 0.14$ & $16.79 \pm 0.19$ \\
$\chi^{2} \ldots \ldots \ldots \ldots \ldots$ & $\ldots$ & $\ldots$ & $\ldots$ & 9.0 \\
$2351-154 \ldots \ldots \ldots$ & 1980 Jul & $>16.88$ & $16.40 \pm 0.17$ & $15.84 \pm 0.13$ \\
& 1981 Oct & $16.25 \pm 0.07$ & $15.59 \pm 0.06$ & $14.67 \pm 0.08$ \\
& 1981 Nov & $16.12 \pm 0.08$ & $15.28 \pm 0.07$ & $14.34 \pm 0.08$ \\
$\chi^{2} \ldots \ldots \ldots \ldots \ldots$ & $\ldots$ & $\ldots$ & 39.9 & 97.2 \\
\hline
\end{tabular}




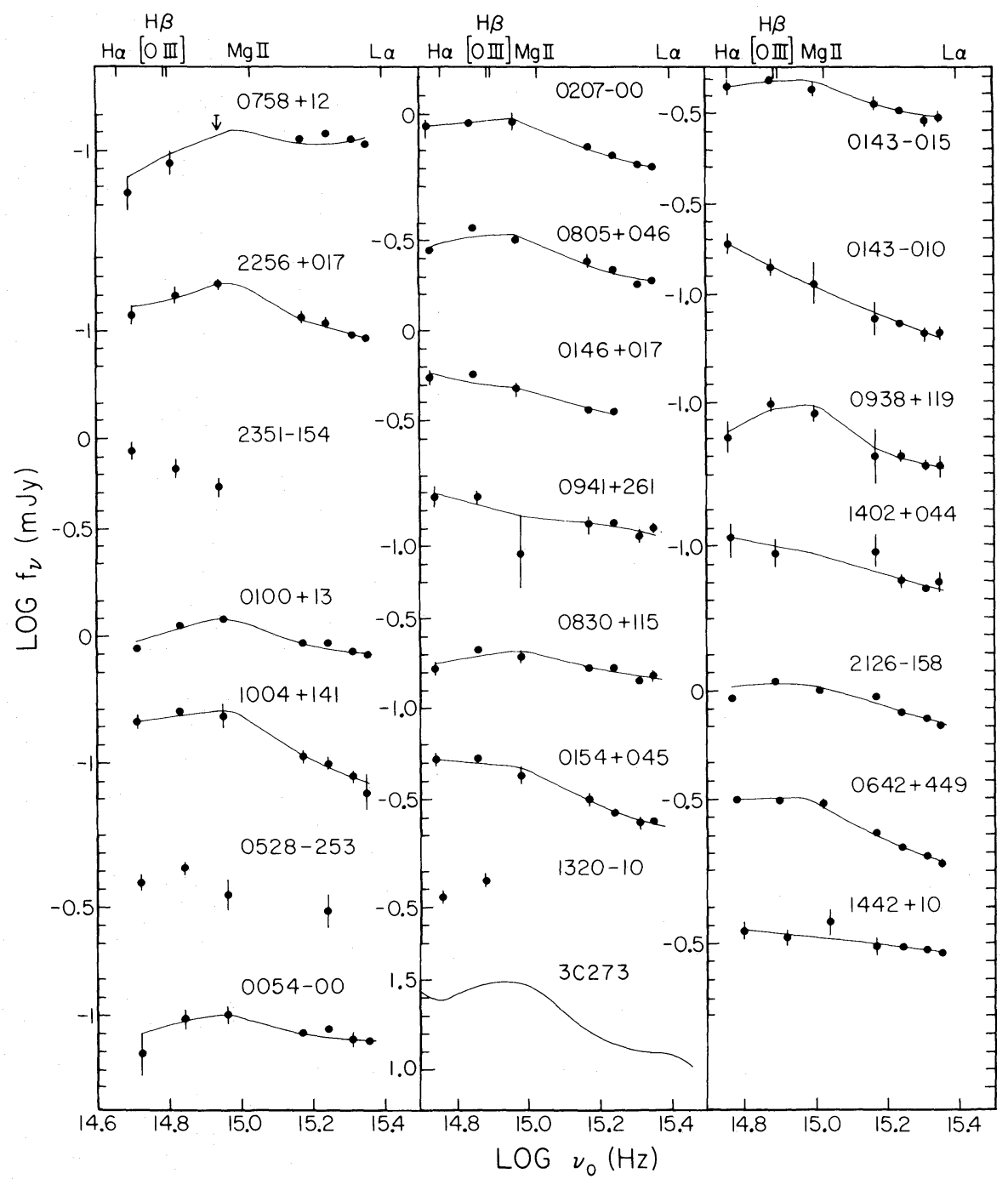

Fig. 1.-The flux densities for high-redshift quasars plotted vs. rest frequency for each object. The infrared observations are corrected, where necessary, for the presence of the emission lines noted at the top of the figure. Included for comparison is the continuum energy distribution of 3C 273. The line through the data for each quasar is the best fit model of a $3000 \AA$ bump and power-law continuum as described in the text.

\section{DISCUSSION}

The quasars observed represent roughly half of those known with redshifts $z \geq 2.66$; thus the results obtained here are probably representative of high-redshift quasars as a whole. The high-redshift nature of the sample means the quasars in the sample will be of intrinsically high luminosity. Indeed, the two highest luminosity objects in this sample, $0100+130$ (PHL 957) and PKS 2126-158, are among the most luminous quasars known. Table 4 contains estimates of the luminosity at rest frame wavelengths of 0.17 and $0.41 \mu \mathrm{m}$. Figure 2 shows histograms of distributions of the luminosities of the present sample. The range in luminosities includes $2.1<L_{U}<34 \times 10^{12} L_{\odot}$ at ultraviolet wavelengths $(0.17 \mu \mathrm{m})$ and $1.2<L_{o}<21 \times 10^{12} L_{\odot}$ at visual wavelengths $(0.41 \mu \mathrm{m})$. In each case the luminosity was approximated by the product $v_{0} F_{v_{0}}$, where $F_{v_{0}}$ is the emitted power density at the rest frequency $v_{0}$. In this discussion, the wavelengths referred to are, unless otherwise specified, quasar rest frame wavelengths, and the continuum spectral indices and luminosities have been calculated using the flux densities corrected for line emission, as previously described. In estimating these luminosities $H_{0}$ was taken to be $50 \mathrm{~km} \mathrm{~s}^{-1} \mathrm{Mpc}^{-1}$ with $q_{0}$ taken to be +1 ; these values were taken for ease of comparison with the results of NOBM.

The observed range in luminosities overlaps the more luminous half of the quasar sample studied by NOBM. The histograms of luminosities (Fig. 2) show, however, that many of the quasars in this sample are comparable to or less luminous than $3 \mathrm{C} 273$, so the sample studied is not exclusively ultra-high-luminosity objects. The 
TABLE 4

Derived Properties of High-Redshift Quasars

\begin{tabular}{|c|c|c|c|c|c|c|c|c|c|}
\hline \multirow[b]{2}{*}{ Object } & \multirow[b]{2}{*}{$\begin{array}{c}\text { Selection } \\
\text { (Optical/Radio) }\end{array}$} & \multirow[b]{2}{*}{$z$} & \multirow[b]{2}{*}{$\alpha_{\text {UO }}$} & \multirow[b]{2}{*}{$f_{B} / f_{C}$} & \multirow[b]{2}{*}{$\chi^{2} / N$} & \multirow[b]{2}{*}{$\alpha_{\mathrm{OX}}$} & \multicolumn{2}{|c|}{$v_{0} F v_{0}$ at $\log v_{0}=$} & \multirow[b]{2}{*}{$L_{U} / L_{O}$} \\
\hline & & & & & & & $\begin{array}{c}15.24 \\
L_{U}\left(10^{12} L_{\odot}\right)\end{array}$ & 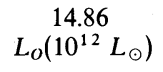 & \\
\hline $0054-00 \ldots \ldots \ldots$ & $\mathbf{R}$ & 2.795 & -0.05 & 0.32 & 1.2 & $<-1.66$ & 2.8 & 1.2 & 2.3 \\
\hline $0100+13 \ldots \ldots \ldots$ & $\mathrm{O}$ & 2.690 & -0.10 & 0.42 & 1.2 & $<-1.66$ & 31.0 & 16.0 & 1.9 \\
\hline $0143-015 \ldots \ldots \ldots$ & $\mathrm{O}$ & 3.14 & -0.27 & 0.29 & 0.5 & $<-1.52$ & 14.0 & 8.6 & 1.6 \\
\hline $0143-010 \ldots \ldots \ldots$ & $\mathrm{O}$ & 3.16 & -0.89 & -0.05 & 0.4 & $<-1.33$ & 2.8 & 2.5 & 1.1 \\
\hline $0146+017 \ldots \ldots \ldots$ & $\mathrm{O}$ & 2.91 & -0.43 & 0.10 & 1.2 & $<-1.63$ & 14.0 & 9.2 & 1.5 \\
\hline $0154+045 \ldots \ldots \ldots$ & $\mathrm{O}$ & 2.99 & -0.57 & 0.29 & 1.0 & $\ldots$ & 10.0 & 8.9 & 1.1 \\
\hline $0207-003 \ldots \ldots \ldots$ & $\mathrm{O}$ & 2.84 & -0.35 & 0.36 & 0.6 & $\ldots$ & 22.0 & 14.0 & 1.6 \\
\hline $0528-25 \ldots \ldots \ldots$ & $\mathbf{R}$ & 2.765 & $\ldots$ & $\ldots$ & $\ldots$ & $\ldots$ & 11.0 & 7.9 & 1.4 \\
\hline $0642+449 \ldots \ldots \ldots$ & $\mathbf{R}$ & 3.402 & -0.56 & 0.36 & 0.5 & -1.19 & 7.7 & 6.0 & 1.3 \\
\hline $0758+12 \ldots \ldots \ldots$ & $\mathbf{R}$ & 2.660 & +0.31 & 0.49 & 4.6 & $\ldots$ & 3.9 & 1.5 & 2.6 \\
\hline $0805+046 \ldots \ldots \ldots$ & $\mathbf{R}$ & 2.877 & -0.31 & 0.42 & 2.9 & -1.39 & 8.1 & 5.9 & 1.4 \\
\hline $0830+115 \ldots \ldots \ldots$ & $\mathbf{R}$ & 2.974 & -0.11 & 0.29 & 2.0 & $<-1.25$ & 6.6 & 3.5 & 1.9 \\
\hline $0938+119 \ldots \ldots \ldots$ & $\mathbf{R}$ & 3.190 & -0.29 & 0.79 & 0.9 & $<-1.26$ & 2.1 & 1.8 & 1.2 \\
\hline $0941+26 \ldots \ldots \ldots$ & $\mathbf{R}$ & 2.910 & -0.35 & -0.10 & 1.4 & $\ldots$ & 5.1 & 3.1 & 1.6 \\
\hline $1004+141 \ldots \ldots \ldots$ & $\mathbf{R}$ & 2.707 & -0.51 & 0.58 & 0.5 & $\ldots$ & 3.4 & 2.9 & 1.2 \\
\hline $1320-10 \ldots \ldots \ldots$ & $\mathrm{O}$ & 3.14 & $\ldots$ & $\ldots$ & $\ldots$ & $\ldots$ & $\ldots$ & 7.9 & $\ldots$ \\
\hline $1402+044 \ldots \ldots \ldots$ & $\mathbf{R}$ & 3.202 & -0.49 & 0.05 & 0.6 & -1.37 & 2.5 & 1.6 & 1.6 \\
\hline $1442+101 \ldots \ldots \ldots$ & $\mathbf{R}$ & 3.53 & -0.23 & 0.00 & 0.5 & -1.38 & 15.0 & 6.9 & 2.2 \\
\hline $2126+158 \ldots \ldots \ldots$ & $\mathbf{R}$ & 3.27 & -0.32 & 0.24 & 3.9 & -1.22 & 34.0 & 21.0 & 1.6 \\
\hline $2256+017 \ldots \ldots \ldots$ & $\mathbf{R}$ & 2.663 & -0.25 & 0.58 & 0.7 & $\ldots$ & 3.6 & 2.3 & 1.6 \\
\hline $2351-15 \ldots \ldots \ldots$ & $\mathbf{R}$ & 2.665 & $\ldots$ & $\ldots$ & $\ldots$ & $\ldots$ & $\ldots$ & 7.7 & $\ldots$ \\
\hline
\end{tabular}

NoTE.-All parameters are calculated with correction made for presence of emission lines in photometric band. Uncertainties are \pm 0.05 in $\alpha_{\text {UO }}$ and \pm 0.1 in $f_{B} / f_{C}$.

visually and radio-selected quasars span the same range of ultraviolet luminosities; this shows that the limiting magnitudes for the visual searches and radio identification programs are quite similar.

Finally, Figure 2 shows the histogram of the ratio $L_{U} / L_{o}$, i.e., the ratio of $L_{U}$, the luminosity at $0.17 \mu \mathrm{m}$, to $L_{o}$, the luminosity at $0.41 \mu \mathrm{m}$. The mean value of this ratio is 1.6 , and in no case is the ratio less than 1.1. Thus, this sample of quasars is indeed a sample of ultraviolet-bright quasars, i.e., quasars which are more luminous at ultraviolet wavelengths than at other observed wavelengths. NOBM have shown that in many low-redshift quasars there is a peak in the power emitted around $3 \mu \mathrm{m}$. It would thus be interesting to study the rest frame infrared properties of the current sample of high-redshift quasars to see if a similar peak occurs in these quasars. Unfortunately, current instrumental sensitivities preclude a significant program of such observations.

The continuum shapes displayed in Figure 1 show a wide variety of behavior that is rarely characterizable by a simple power law. Many show a maximum in the continuum between 0.3 and $0.4 \mu \mathrm{m}$ and have a general convex energy distribution with the slope steepening with increasing frequency. The variety in the observed continua is quite similar to that found by NOBM in the sample of low-redshift quasars.

We have attempted to fit the continuum flux densities as the sum of a power law, $f_{v} \propto v^{\alpha}$, and a "bump" centered at $0.31 \mu \mathrm{m}$ which represents the $3000 \AA$ excess that has been previously studied by several authors (e.g., Baldwin 1975; Grandi and Phillips 1980; NOBM;
Puetter et al. 1982; Grandi 1982; Malkan and Sargent 1982). For this fitting, the multichannel continuum flux densities at rest frequencies corresponding to $\log v_{0}=$ $15.17,15.24,15.31$, and 15.35 and the infrared observations corrected for the presence of the strongest emission lines were used; the multichannel frequencies were chosen so the continua were free of emission lines. The adopted shape of the $3000 \AA$ bump was derived from the continuum energy distribution of $3 \mathrm{C} 273$ as reported by Boggess et al. (1979) by assuming the bump was the excess flux over the power law fitted between $5586 \AA$ and $1600 \AA$. The amplitude of the $3000 \AA$ excess is described by the ratio of $f_{B}$, the flux density of the bump, at its peak wavelength, $0.31 \mu \mathrm{m}$, to $f_{C}$, the flux density of the power-law component at the same wavelength. This $3000 \AA$ bump is clearly the sum of components that have been studied in detail by several authors (e.g., Grandi 1982; Puetter et al. 1982; Malkan and Sargent 1982; Oke, Shields, and Korycansky 1982). The adopted shape agrees well with that found by Oke, Shields, and Korycanski (1982). The best fit parameters of $\alpha_{U O}$ and of $f_{B} / f_{C}$ are given in Table 4 , and histograms of their distributions are given in Figure 3. The uncertainties in the model parameters are roughly \pm 0.05 in $\alpha_{\text {UO }}$ and \pm 0.1 in $f_{B} / f_{C}$.

Eighteen quasars have enough observations to be fitted. For these as a group, the power law plus bump gave a vastly superior fit than the fit with a power law only. For 15 quasars, the model gave statistically satisfactory fits to the observations. For 2126-158, $0805+046$, and $0758+12$, no simple model gave a satisfactory fit to the observations. In the two cases where 

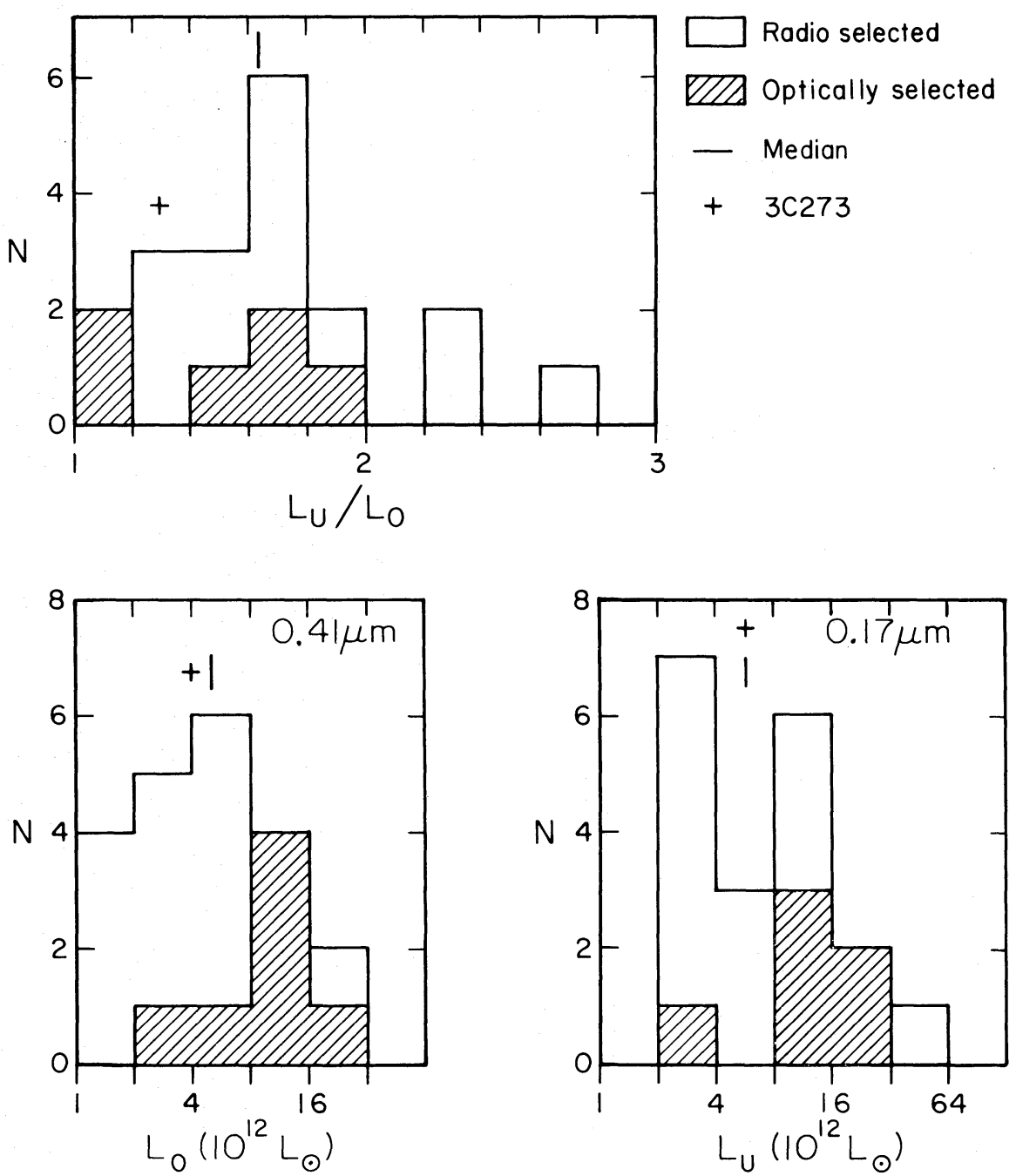

Fig. 2.-Histograms of the luminosities and the ratio of luminosities of the sample of quasars at ultraviolet and visual wavelengths. The visually and radio-selected quasars are indicated separately, as is the median of the entire sample. The value of the appropriate quantity for $3 \mathrm{C} 273$ is also shown in the plots.

the model formally resulted in negative amplitudes for the bump, the bump amplitudes were consistent within the uncertainties with zero amplitude. The success of the simple model suggests that at the resolution of these observations a more complex spectral structure is not required to describe the visual/ultraviolet continua of these quasars.

The mean spectral index from visual through ultraviolet wavelengths of this sample of high-redshift quasars is $-0.37 \pm 0.05$. This mean spectral index is in good agreement with the spectral index $\alpha=-0.5 \pm 0.1$ found by Richstone and Schmidt (1980) for a large sample of quasars with a large range of redshifts. The mean bump to continuum ratio $f_{B} / f_{C}$ is 0.30 with a dispersion of 0.20 .

From the data of Boggess et al. (1979), the spectral index $\alpha_{U}$ of $3 C 273$ is $-0.54 \pm 0.05$, and the bump to continuum ratio $f_{B} / f_{C}$ is $0.7 \pm 0.1$. The spectral index of 3C 273 is well within the range of $\alpha_{\mathrm{UO}}$ found for the high-redshift quasars, while the strength of the bump in 3C 273 is somewhat greater than the mean bump amplitude although within the range found for the highredshift sample. The success of the model fits, using the bump excess defined from the 3C 273 data, and the fact that the range of parameters derived from the highredshift samples encompasses $3 C 273$ argue that at this resolution the ultraviolet/visual continua of the highredshift quasars are similar to that of $3 \mathrm{C} 273$, and by inference to the continua of other low-redshift quasars, since 3C 273 is archetypical of this class of objects.

Oke and Korycansky (1982) have defined a spectral index $\alpha_{\text {UO }}$ between 2000 and $1200 \AA$ from the observations of many of the quasars of this sample. They find a mean value $\alpha_{U O}=-0.49 \pm 0.04$. There is thus a suggestion of a steepening of the power-law continuum at shorter wavelengths. This marginal steepening is 


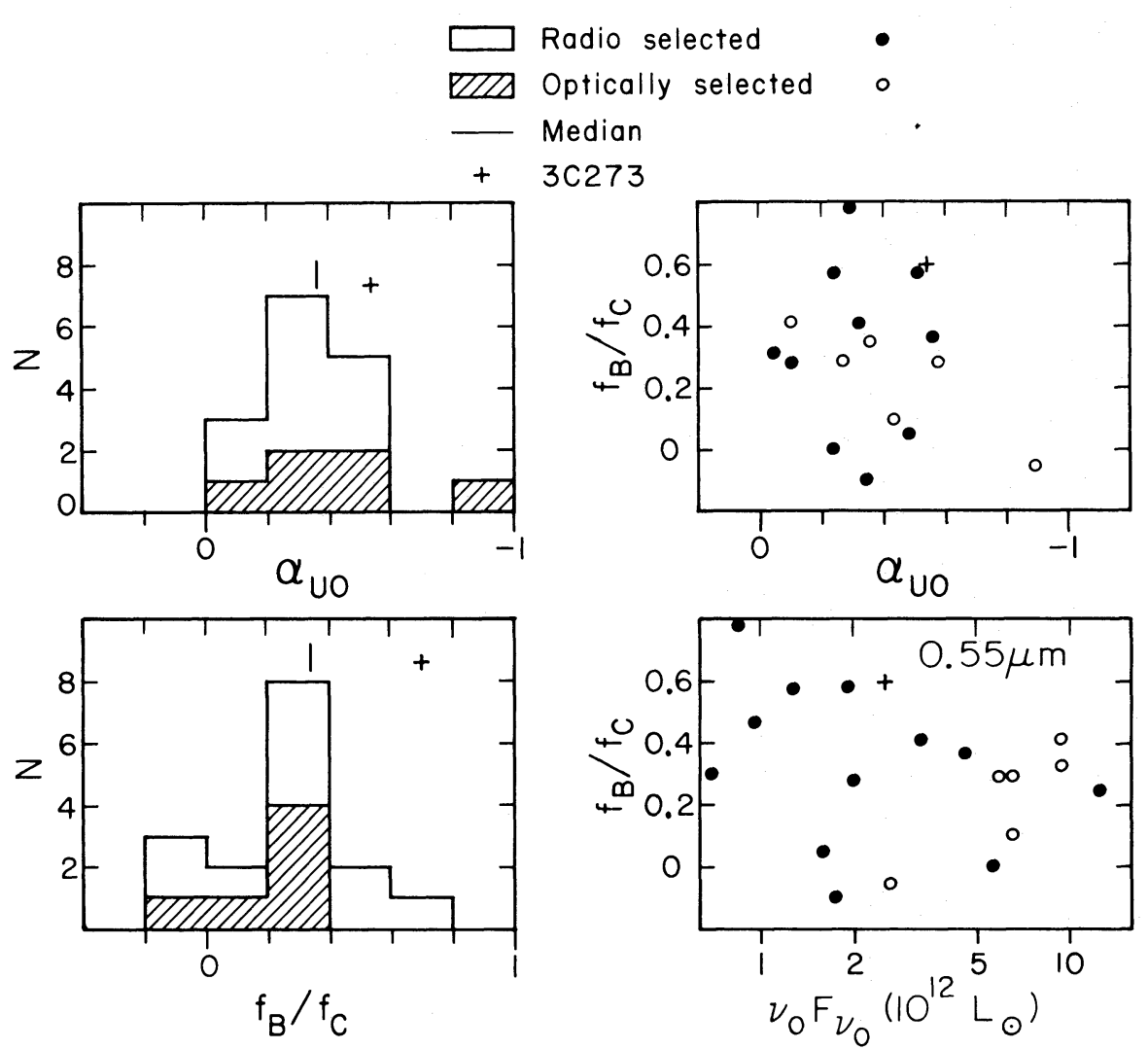

FIG. 3. - The histograms of $\alpha_{\mathrm{UO}}$, the spectral index, and of $f_{B} / f_{C}$, the ratio of the bump flux density to the power-law continuum flux density at $0.31 \mu \mathrm{m}$ for the high-redshift quasars. The visually and radio-selected quasars are indicated separately, and the values of these quantities for the median of the entire sample and for $3 \mathrm{C} 273$ are shown in the figure. Also plotted are the ratio $f_{B} / f_{C}$ vs. spectral index $\alpha_{U O}$ and $f_{B} / f_{C}$ vs. $v_{0} F_{v_{0}}$, the luminosity at $0.55 \mu \mathrm{m}$ for the observed quasars. The visually and radio-selected quasars are indicated by separate symbols, as is $3 \mathrm{C} 273$.

significantly less than that derived by Cheney and Rowan-Robinson (1981) from an analysis of the HewittBurbidge catalog (1980).

A search was made for correlations between the various parameters of the model fit. None was found. In particular, as shown in Figure 3, there is no correlation between the power-law slope and the strength of the bump. It is also seen in Figure 3 that there is no obvious correlation of bump excess with luminosity, although Malkan and Sargent (1982) have suggested that the strength of the $3000 \AA$ bump is correlated with luminosity for quasars and other active galactic nuclei.

Eleven of the quasars observed in the present sample have been observed with the Einstein Observatory at $\mathrm{X}$-ray wavelengths by Zamorani et al. (1981) and $\mathrm{Ku}$, Helfand, and Lucy (1980); five of these, all radio-selected quasars, were detected at X-ray wavelengths. For the latter sample of five quasars, there is no correlation between $\alpha_{\mathrm{UO}}$ and $\alpha_{\mathrm{OX}}$, the visual/X-ray spectral index. The mean $\alpha_{\text {ox }}$ of these five quasars is -1.31 , while the mean $\alpha_{\mathrm{UO}}$ of the same quasars is -0.35 . Clearly, a significant steepening of the quasar spectra must occur at wavelengths shortward of the observed ultraviolet wavelengths. For two cases where high-resolution spectra are available, Oke and Korycansky (1982) showed that the steepening did not begin at least until the Lyman jump was reached. International Ultraviolet Explorer (IUE) observations of the high-redshift quasar Q2204-408 by Wilson, Carnochan, and Gondhalekar (1979) give a spectral index between 1500 and $400 \AA$ of -1.5 , which suggests a steepening slope below $900 \AA$. Observations of PG $1115+080$ with $I U E$ by Green et al. (1980) show a similar effect.

There is no evidence in these observations for different continuum properties among the visually and radio-selected quasars in the sample. This is illustrated by the histograms of Figure 3, which show that both visually and radio-selected quasars span the full range of the spectral index, $\alpha_{U O}$, and of the bump to continuum ratio $f_{B} / f_{C}$. Thus, to the level at which the visual identification of the radio sample did not introduce a bias in the visual properties of the radio sample, no differences exist in the continuum properties at ultraviolet and visual wavelengths between the visually and radio-selected quasars observed here.

\section{SUMMARY}

A photometric/spectrophotometric study of 21 highredshift quasars in the observed wavelength range 
$0.3-2.2 \mu \mathrm{m}$ (rest wavelength range $\sim 800-5500 \AA$ ) has led to the following conclusions:

1. The visual/ultraviolet continuum distributions of the high-redshift quasars are fitted adequately by a sum of a power-law continuum and a $3000 \AA$ bump excess similar in shape to that found in 3C 273.

2. Overall, the high-redshift quasars in this sample have very similar rest frame visual/ultraviolet continuum properties which are similar to those of the archetypical quasar 3C 273.

3. There is no correlation apparent between the visual/ultraviolet spectral index and the visual/X-ray spectral index for the five high-redshift quasars detected at X-ray wavelengths.

4. The visual/ultraviolet continuum properties of this sample of quasars show no correlations with selection technique (i.e., radio versus visual selection).

We thank J. Carrasco and K. Sellgren for help with the observations. Infrared research at Caltech is supported by grants from NSF and NASA. The research of J. B. O. is supported by NASA grant NSG 05-002-134

\section{REFERENCES}

Baldwin, J. A. 1975, Ap. J., 201, 26

Baldwin, J. A., Burke, W. L., Gaskell, C. M., and Wampler, E. J. 1978, Nature, 273, 431

Boggess, A., et al. 1979, Ap. J. (Letters), 230, L131.

Cheney, J. E., and Rowan-Robinson, M. 1981, M.N.R.A.S., 195, 831.

Grandi, S. A. 1982, Ap. J., 255, 25 .

Grandi, S. A., and Phillips, M. M. 1980, Ap. J., 239, 475.

Green, R. F., Pier, J. R., Schmidt, M., Estabrook, F. B., Lane, A. L. and Wahlquist, H. D. 1980, Ap. J., 239, 483.

Hewitt, A., and Burbidge, G. 1980, Ap. J. Suppl., 43, 57.

$\mathrm{Ku}$, W., Hefland, D., and Lucy, L. 1980, Nature, 288, 323.

Kunth, D., Sargent, W. L. W., and Kowal, C. 1981, Astr. Ap. Suppl., 44, 229.

MacAlpine, G. M., and Lewis, D. W. 1978, Ap. J. Suppl., 36, 587.

MacAlpine, G. M., Smith, S. B., and Lewis, D. W. 1977, Ap. J. Suppl., 35, 197.
Malkan, M., and Sargent, W. L. W. 1982, Ap. J., 254, 22.

Neugebauer, G., Oke, J. B., Becklin, E. E., and Matthews, K. 1979, Ap. J., 230, 79 (NOBM).

Oke, J. B., and Korycansky, D. G. 1982, Ap. J., 255, 11

Oke, J. B., Shields, G. A., and Korycansky, D. G. 1982, in preparation. Puetter, R. C., Burbidge, E. M., Smith, H. E., and Stein, W. A. 1982 Ap. J., 257, 487.

Puetter, R. C., Smith, H. E., Willner, S. P., and Pipher, J. L. 1981, Ap. J., 243, 345.

Richstone, D. D., and Schmidt, M. 1980, Ap. J., 235, 377

Smith, H. E., Jura, M., and Margon, B. 1979, Ap. J., 228, 369.

Soifer, B. T., Neugebauer, G., Oke, J. B., and Matthews, K. 1981, Ap. J., 243, 369.

Wilson, R., Carnochan, D. J., and Gondhalekar, P. M. 1979, Nature, 277, 457.

Zamorani, G., et al. 1981, Ap. J., 245, 357

J. B. OKe: Division of Physics, Mathematics, and Astronomy, Robinson Laboratory 105-24, California Institute of Technology, Pasadena, CA 91125

J. H. Lacy, K. Matthews, G. Neugebauer, and B. T. Soifer: Division of Physics, Mathematics, and Astronomy, Downs Laboratory 320-47, California Institute of Technology, Pasadena, CA 91125 